\title{
Über Protamine und Histone. ${ }^{1}$ ).
}

\author{
Von
}

A. Kossel und H. Pringle.

(Aus dem physiologischen Institut in Heidelberg.)

Den Feststellungen über die Natur und die Menge der einzelnen im Eiweiß vorhandenen "Bausteine» werden Untersuchungen über die Anordnung dieser Gruppen in dem ganzen Molekül folgen müssen. Der nächste Weg, um zu Ergebnissen dieser Art zu gelangen, bietet sich in dem stufenweisen Abbau der Eiweißsubstanzen. Natürlich wird man um so mehr Aussicht auf sichere Ergebnisse haben, je einfacher der untersuchte Eiweißkörper gebaut ist, und deshalb haben wir die folgenden Untersuchungen zunächst auf ein einfaches Protamin, das Clupein, gerichtet. Aber auch bei komplizierteren Eiweißsubstanzen wird man mit der heutigen Methodik durch einen stufenweisen Abbau wertvolle Resultate erzielen können, indem man einfacher gebaute Gruppen aus dem ganzen Molekül herauslöst und deren Konstitution zu erforschen sucht. Wir haben im Vergleich mit dem Protamin diejenigen Eiweißkörper untersucht, welche den Protaminen am nächsten stehen, nämlich die Histone, und es ist in der Tat möglich gewesen, aus ihnen einen peptonartigen Körper zu gewinnen, der weniger Bausteine enthält, wie die meisten Eiweißsubstanzen, und der als Ausgangsmaterial für weitere Konstitutionsforschungen dienen soll.

Bekanntlich müssen wir auch bei den einfachsten Eiweißstoffen immer mit der Möglichkeit rechnen, daß die Substanzen, welche wir unter den Händen haben, aus einer gewissen Zahl einzelner einander ähnlicher Körper zusammengesetzt sind.

1) Der auf das Clupein bezügliche Teil dieser Mitteilung wurde am 3. August 1906 in der chemischen Sektion der Association française pour l'avancement des sciences in Lyon von A. Kossel vorgetragen und im Journal de l'association amicale des étudiants en pharmacie de Lyon, Jahrg. 1906, S. 65, vorläufig publiziert.

Hoppe-Seyler's Zeitschrift f. physiol. Chemie. XLIX. 
Diese Unsicherheit hat aber nicht verhindert, daß wir über den chemischen Bau der Protamine ganz bestimmte Vorstellungen gewinnen und uns ein allgemeines Bild von der Anordnung der Bausteine machen können, wie es bei den übrigen, komplizierter gebauten Eiweißsubstanzen noch nicht möglich gewesen ist.

Das Clupein, welches für die ersten Untersuchungen diente, gehört einer Gruppe von Protaminen an, die wir nach den bestbekannten Repräsentanten als «Salmingruppe * bezeichnen können. Diese Gruppe umfaßt außer dem Clupein noch das Salmin und Scombrin. Die Zusammengehörigkeit dieser Körper wird erwiesen vor allem durch das quantitative Verhältnis zwischen ihren Bausteinen, welches aus folgender $\mathrm{Zu}$ sammenstellung ersichtlich ist.

Argininstickstoff in Prozenten des Gesamtstickstoffs.

$\begin{array}{lllll}\text { Salmin } & \left.87,8^{1}\right) & \left.89,2^{2}\right) & & \\ \text { Clupein } & \left.88,0^{3}\right) & \left.89,1^{4}\right) & \left.88,7^{4}\right) & \left.87,9^{4}\right) \\ \text { Scombrin } & \left.88,9^{5}\right) & \left.88,95^{5}\right) & & \end{array}$

Ein Teil der früher publizierten Bestimmungen hatte wohl wegen der damals noch unvollkommenen Ausbildung der Methoden - etwas niedrigere Zahlen ergeben.

Somit ist 8/9 des gesamten Stickstoffs in Form von Arginin nachweisbar, der Rest als Alanin, Serin, Amidovaleriansäure oder Prolin. Diese Monoamidosäuren treten aber nicht alle gleichzeitig neben einander auf, sondern jedes der genannten Protamine enthält 2 oder 3 derselben neben dem Arginin.

Nach Feststellung dieser Zahlen haben wir die bereits früher von M. Goto im hiesigen Institut untersuchte Frage nach dem Verhältnis der Protone zu den Protaminen wieder aufgenommen. Sind die Protone, welche als nächste hydrolytische Zersetzungsprodukte aus dem Clupein hervorgehen, unter einander ungleichartig oder gleichartig? Die von Goto an verschie-

1) A. Kossel und F. Kutscher, Diese Zeitschrift, Bd. XXXI, S. 181 (1900).

2) A. Kossel und H. D. Dakin, Diese Zeitschrift, Bd. XLI, S. 413 (1904).

3) A. Kossel und H. D. Dakin, Nicht publiziert.

4) H. Pringle, siehe unten.

5) A. Kossel, siehe unten. 
denen Fraktionen des Protongemisches ausgeführten Elementaranalysen beweisen, dab dies Gemisch aus verschiedenartigen Stoffen zusammengesetzt ist, aber die unten mitgeteilten quantitativen Spaltungsversuche zeigen, $d a ß$ diese Protone, ebenso wie ihre Muttersubstanz das Clupein, $8 / 9$ des Stickstoffs in Form von Arginin enthalten. Die folgende Tabelle, welche die weiter unten beschriebenen Versuche des einen von uns (H. Pringle) enthält, läßt dies deutlich erkennen.

Argininstickstoff in Prozenten des Gesamtstickstoffs der aus Clupein gewonnenen Protone.

$\begin{array}{ccc}\text { Fraktion } & \text { Ia (Versuch V) } 87,6 \\ * & \text { Ib }(* & \text { ) } 87,2 \\ * & \text { II }(* & \text { ) } 88,8 \\ * & \text { III }(* & \text { ) } 87,0\end{array}$

Proton aus Versuch IV 88,12

Da das Arginin 4 Atome Stickstoff enthält, so ist im Proton und im Protamin auf je 2 Argininmoleküle 1 Molekül einer Monoamidosäure enthalten.

Nach den bisherigen Erfahrungen und unsern heutigen Anschauungen über die Verkettung der Gruppen ergeben sich keine anderen Möglichkeiten der Kombination, als solche, bei denen mindestens zwei Arginingruppen mit einander verbunden sind, wir haben also "Diarginid* (*Diarginyl*) oder «Polyarginid -Gruppen in dem Protamin anzunehmen.

Die Verkettung der Monoamidosäuren kann eine symmetrische oder unsymmetrische sein, insofern entweder eine regelmäßig sich wiederholende Reihenfolge des Diarginids und der Monoamidokörper oder eine unregelmäßige Anhäufung der Gruppen in einem Teile des Moleküls vorhanden ist. Bezeichnet man die Arginingruppen mit a, die Monoamidosäuren im allgemeinen mit $b$ und die verschiedenen Monoamidosäuren mit $b^{\prime}, b^{\prime \prime}, b^{\prime \prime \prime}$, so kann man durch folgende Formeln zwei beliebig gewählte Beispiele für eine symmetrische und eine unsymmetrische Anordnung darstellen.

Symmetrische Anordnung: $a a b / a a b^{/ / a a b} / 1 /$

Unsymmetrische Anordnung: aagaaab $b^{/} b^{/ /} b^{\prime \prime \prime}$. 
Die folgenden Untersuchungen machen es im höchsten Grade wahrscheinlich, daß das Clupein dem symmetrischen Typus angehört, wenn es in seiner Konstitution auch nicht gerade dem hier aufgeführten Beispiel zu entsprechen braucht. Selbstverständlich würde eine andere symmetrische Anordnung z. B. ${ }^{\text {b }}$ a ba aa ba $^{\prime \prime \prime}$ ebensowenig ausgeschlossen sein, wie eine kleine Abweichung vom symmetrischen Bau, die nur einzelne Stellen einer längeren Reihe z. B. den Anfang oder das Ende derselben betrifft.

Die Annahme einer symmetrischen Struktur in diesem Sinne stimmt auch mit den schon früher publizierten Ergebnissen überein, welche M. Goto im hiesigen Institut bei der Untersuchung der Protone gewonnen hat, und welche sich am einfachsten erklären lassen, wenn man den Protonen nach obiger Ausdrucksweise die Formeln aab oder aba oder baa zuschreibt, d. h. wenn man sie als Verbindungen von zwei Argininmolekülen mit einem Molekül einer Monoamidosäure auflaßt.

Goto fand nämlich für das Molekulargewicht der von ihm mit großer Sorgfalt gereinigten Clupeonpräparate folgende Zahlen:

nach der Siedemethode im Mittel: 419,2, nach der Gefriermethode im Mittel: 423,7.

Nun sind im Clupein bisher folgende Monoamidokörper gefunden worden: Alanin, Serin, Prolin und Amidovaleriansäure. Für dies Schema aab (oder aba oder baa) berechnen sich hiernach folgende Molekulargewichte:

$\begin{array}{lr}\text { Diarginylalanin und Isomere: } M=401 \\ \text { Diarginylserin usw. } & 417 \\ \text { Diarginylprolin usw. } & 427 \\ \text { Diarginylamidovaleriansäure usw. } & 429 .\end{array}$

Es ist schwer denkbar, daß die Übereinstimmung dieser Zahlen mit den von M. Goto gefundenen einem bloßen Zufall zuzuschreiben ist, und wir halten es für wahrscheinlich, daß die als Protone bezeichneten Körper wirklich Gemische sind, welche die genannten Arginide enthalten. 
Hiermit stimmen nicht nur die Argininbestimmungen, sondern auch einige der von Goto ausgeführten Elementaranalysen. Dieser Forscher fand bei den verschiedenen Fraktionen, welche er aus dem Protongemisch darstellte, verschiedene Werte für $\mathrm{C}, \mathrm{H}$ und $\mathrm{N}$. Einige derselben sind noch unverständlich, ${ }^{1}$ ) andere lassen sich mit obiger Annahme in Einklang bringen. Z. B. analysierte Go to eine Kupferverbindung des Protons, deren Zahlen nach Ersatz des Kupfers durch Wasserstoff der Formel $\mathrm{C}_{17} \mathrm{H}_{85} \mathrm{~N}_{8} \mathrm{O}_{15}$ entsprachen: ${ }^{2}$ )

$\begin{array}{ccc} & \text { Gefunden: } & \text { Berechnet für } \mathrm{C}_{17} \mathrm{H}_{35} \mathrm{~N}_{9} \mathrm{O}_{15} \text { : } \\ \mathrm{C} & 45,92 \% & 45,84 \% \\ \mathrm{H} & 7,8 \% & 7,86 \% \\ \mathrm{~N} & 28,0 \% & \%\end{array}$

Nimmt man an, daß 2 Moleküle Arginin und 1 Molekül Prolin unter Austritt von $2 \mathrm{H}_{2} \mathrm{O}$ vereinigt sind, so erhält man die Formel $\mathrm{C}_{17} \mathrm{H}_{33} \mathrm{~N}_{9} \mathrm{O}_{14}$, deren Hydrat die obige Formel ist. Auch mit der Annahme der entsprechenden Verbindung von Arginin und Amidovaleriansäure läßt sich die Formel in Einklang bringen, wenn man eine andere Bindungsweise des Kupfers annimmt. Immerhin ist die Reingewinnung einzelner chemischer Individuen aus dem Protongemisch noch nicht sicher herbeizuführen und es ist uns trotz mehrfacher Versuche nicht gelungen, ein Protonpräparat darzustellen, welches nur eine oder auch nur vorwiegend eine Amidosäure enthält.

Ein bemerkenswertes Resultat ergab die Einwirkung der salpetrigen Säure auf das Protongemisch. Hierbei entstand ein «Desamidoproton», welches bei der Hydrolyse durch Säuren direkt Ornithin abspaltet. Es ist also durch die salpetrige Säure eine Guanidingruppe zerstört oder wenigstens so verändert worden, daß ihr Rest bei der nachfolgenden Hydrolyse von der Ornithingruppe abgespalten wird. Diese Tatsache ist am leichtesten zu erklären, wenn man den Typus aab oder $\mathrm{aba}^{3}$ ) für eine im Protongemisch enthaltene Substanz annimmt (z. B. Diarginylvalin oder Arginylvalylarginin). Bei Annahme des Typus baa (z. B. Valylarginylarginin) wäre die Erklärung weniger naheliegend.

Diese von A. Kossel schon früher mitgeteilte ${ }^{4}$ ) Ein-

1) Z. B. die in der Abhandlung von Goto (Diese Zeitschrift, Bd. XXXVII, S. 94) publizierten Analysen.

2) Noch nicht publizierte Analyse des Herrn M. Goto.

s) Wobei die das freie Carboxyl tragende Gruppe an die rechte Seite des Ausdrucks gesetzt ist.

$\left.{ }^{4}\right)$ cf. Biochemisches Zentralblatt, Bd. V, S. 7, März 1906. 
wirkung der salpetrigen Säure muß wohl zur Erklärung einer neuerdings von Skraup und Hoernes beobachteten ${ }^{1}$ ) Tatsache herangezogen werden. Diese Forscher fanden nämlich, daß das durch salpetrige Säure aus dem Casein dargestellte Desamidocasein bei der Hydrolyse durch Säuren nur geringe Mengen Arginin liefert. Die Arginingruppe wird also auch hier durch salpetrige Säure teilweise zerstört und es ist wohl wahrscheinlich, da $\ddot{B}$ auch in diesem Falle eine gewisse Menge Ornithin an Stelle des fehlenden Arginins abgespalten wird.

Eine ganz analoge Umwandlung des Protamins oder Protons beobachteten A. Kossel und H. D. Dakin unter anderen Verhältnissen. ${ }^{2}$ ) Läßt man Darmextrakt bei Gegenwart von antiseptischen Stoffen längere Zeit auf Protamin einwirken, so bildet sich ein « $\beta$-Clupeon» genanntes Zersetzungsprodukt, welches im wesentlichen die Eigenschaften der Protone besitzt. Dasselbe liefert bei der Hydrolyse mit Säuren weniger Arginin als das Proton, und an Stelle des fehlenden Arginins eine nicht unbeträchtliche Menge Ornithin. Die Ursache dieser Einwirkung ist aber nicht bekannt, vermutlich beruht sie auf der Gegenwart eines Enzyms (Oxydase?). -

Diese Resultate, so fragmentarisch sie auch sind, geben doch eine annähernde Vorstellung von dem Bau der einfachsten Eiweißkörper. Auch dürfte sich aus ihnen manche Anregung für das Studium der komplizierteren Eiweißkörper ergeben. Besonders naheliegend ist die Frage, ob und in welchem Umfange protonartige Aggregate und Diarginylgruppen auch in dem Molekül der typischen Eiweißkörper vorkommen. Bei der Bildung der Protamine im Organismus der Fische müssen diese einfach und regelmäßig gebauten Protaminketten aus den verwickelten Strukturen der höheren Eiweißkörper hervorgehen. ${ }^{3}$ )

Wenn man bei den komplizierteren Eiweißkörpern Analogien mit dem Bau der Protamine aufsuchen will, so wird man sich zunächst zu den Histonen wenden müssen, denn diese

1) Sitzungsber. d. Akademie d. Wissensch. in Wien. Math.-naturw. KIasse, Bd. CXV, Abt. IIb, Juni 1906.

2) Diese Zeitschrift, Bd. XLII, S. 185 (1904).

3) cf. Diese Zeitschrift, Bd. XLIV, S. 347. 
stehen, wie die Untersuchungen von A. Koss el und F. Kutscher erwiesen haben, ${ }^{1}$ ) den Protaminen am nächsten. Sie bilden eine ziemlich scharf charakterisierte Gruppe, besonders hat sich in der Zusammensetzung der Histone, die aus verschiedenen Organen und von verschiedenen Tierspecies stammen, eine recht bemerkenswerte Übereinstimmung gezeigt. Dies mag durch folgende Zusammenstellung erläutert werden.

Prozente des Gesamtstickstoffs.

\begin{tabular}{|c|c|c|c|c|c|}
\hline & $\begin{array}{c}\text { Histi- } \\
\text { din }\end{array}$ & $\begin{array}{c}\text { Argi- } \\
\operatorname{nin}\end{array}$ & Lysin & $\begin{array}{c}\text { Am- } \\
\text { moniak }\end{array}$ & \\
\hline Histon aus Thymus . . & 1,8 & 25,2 & 8,0 & 7,5 & A. Kossel und \\
\hline Testikeln v. Gadus & 3,3 & 26,8 & 8,5 & 1,7 & (F. Kutscher ${ }^{1}$ ) \\
\hline , Lota . . . & 4,1 & 23,4 & 3,7 & 3,3 & Ehrström ${ }^{2}$ ) \\
\hline - Centrophorus & 4,5 & 25,4 & 7,1 & 1,7 & A. Kossel ${ }^{3}$ ) \\
\hline
\end{tabular}

Es ist bemerkenswert, daß wir bisher keinen Eiweißkörper kennen, welcher die charakteristischen Reaktionen der Histone gibt, ohne den hohen Basengehalt zu besitzen. Andererseits kennen wir auch bisher keinen komplizierteren Eiweißkörper, welcher gleichzeitig ebenso viel Arginin und Lysin liefert, wie die Histone. Eiweißkörper aus Pflanzensamen ergeben zwar einen ebenso großen Arginingehalt, ${ }^{4}$ ) aber weniger Lysin, das Hefeeiweiß soll mehr Lysin liefern, aber weniger Arginin. ${ }^{5}$ ) Bestimmungen des Basenstickstoffs, welche mit unzureichenden Methoden (z. B. der sog. „Hausmannschen Methode") ausgeführt sind, können selbstverständlich nicht zur Beurteilung dieser Verhältnisse dienen.

Von den typischen Histonen sind bisher folgende näher untersucht: 1. Histon aus den Erythrocyten des Vogelbluts, 2. aus

1) Diese Zeitschrift, Bd. XXXI, S. 165.

2) Diese Zeitschrift, Bd. XXXII, S. 350.

3) Noch nicht publiziert.

4) Vgl. A. Kossel und A. J. Patten, Diese Zeitschrift, Bd. XXXVIII, S. 39. Ferner E. Schulze und E. Winterstein, Diese Zeitschrift, Bd. XXXIII, 'S. 547.

5) R. Schröder, Beiträge z. chem. Physiologie und Pathologie, Bd. II, S. 389. 
den Spermatozoen von Gadus, 3. aus der Thymusdrüse. Nicht ganz typisch verhält sich das Lotahiston, ${ }^{1}$ ) immerhin muß man es zu den Histonen rechnen. Das von Bang unter dem Namen Scombron ${ }^{2}$ ) aus den unreifen Testikeln der Makrele dargestellte Präparat weicht in mancher Hinsicht von den Histonen ab und es ist aus der kurzen Beschreibung der Darstellung nicht ersichtlich, in welcher Weise das etwa vorhandene Protamin abgetrennt ist. Das Globin kann nicht als ein Histon bezeichnet werden (cf. unten).

Die folgenden Untersuchungen zeigen nun, daß aus den Histonen durch Pepsinverdauung ein Körper „Histopepton“ abgespalten wird, welcher denselben Anteil des Gesamtstickstoffs in Form von Arginin enthält, wie das ursprüngliche Histon, nämlich 27,2\% (Präp. B) und welcher auch im übrigen in seiner Zusammensetzung manche Ähnlichkeit mit dem Histon zeigt.

Diese Tatsache darf natürlich nur in dem Fall einen Anspruch auf allgemeineres Interesse machen, wenn die Darstellung des Histopeptons eine Gewähr dafür bietet, daß man als „Histopepton" nicht den mittleren Durchschnitt des Gemisches der Verdauungsprodukte, sondern eine bestimmt gekennzeichnete Fraktion oder gar ein chemisches Individuum dargestellt hat. Wir haben uns daher bemüht, eine solche Darstellungsmethode ausfindig $\mathrm{zu}$ machen, welche nur einen bestimmten Teil der Verdauungsprodukte heraussondert, und haben hierzu das Natriumpikrat angewandt, welches die gewöhnlich als Albumosen und Peptone bezeichneten Substanzen in neutraler Lösung nicht fällt. Außerdem wurde das Silberbarytverfahren zur Isolierung des Histopeptons benutzt.

Die Auflösung des Histons durch den Magensaft erfolgt aber nicht in der Weise, daß lauter gleichartige Spaltungsprodukte entstehen. Es folgt dies schon aus der Tatsache, daß der Stickstoffgehalt des Histopeptons ungefähr $20 \%$ beträgt, somit höher ist, als der der typischen Histone (18-18,6\%), ferner aus dem Fehlen der ammoniakbildenden Gruppe im Molekül des Histopeptons - beim Histon ist diese Gruppe bisher stets ge-

1) Diese Zeitschrift, Bd. XXXII, S. 350.

2) Diese Zeitschrift, Bd. XXVII, S. 467. 
funden worden. Einzelne Reaktionen fehlen dem Histon völlig oder sind so schwach, daß man die sie bedingende Atomgruppe nicht zum Bestande des Histonmoleküls rechnen kann. Diese sind: die Huminbildung (sofern man darunter die Bildung braungefärbter Produkte unter der Einwirkung siedender verdünnter Mineralsäuren versteht), die Indolreaktionen und die Schwefelbleireaktion. Diese fehlen selbstverständlich auch dem Histopepton. Die Menge der Monoamidosäuren ist relativ gering, im Filtrat des Phosphorwolframsäureniederschlages (nach der Spaltung mit Schwefelsäure) fand sich nur noch $27,7 \%$ des gesamten Stickstoffs vor.

Es liegt somit im Histopepton eine Kombination von Bausteinen vor, welche sich von der in den übrigen Eiweißkörpern vorhandenen durch eine geringere Menge und Mannigfaltigkeit der Monoamidogruppen und durch das Ü̉berwiegen des Arginins und Lysins sehr deutlich unterscheidet.

Freilich muß man beim Histopepton, ebenso wie bei allen bisher untersuchten peptonartigen Stoffen mit der Möglichheit rechnen, daß diese Substanz später vielleicht als ein Gemisch mehrerer peptonartiger Stoffe erkannt wird. Sollte dies aber geschehen, so muß man erwarten, daß mindestens eine der Komponenten die chemischen Eigentümlichkeiten des Histopeptons (bedeutender Gehalt an basischen Bestandteilen) in noch höherem Maße besitzt als das Histopepton. Jedenfalls ist also in dem Histopepton ein bisher unbekannter Typus peptonartiger Stoffe gekennzeichnet. Eine Beimengung von Arginin, Lysin oder Monoamidosäuren ist durch die Darstellungsweise des Histopeptons ausgeschlossen.

Die Untersuchungen über die Verbreitung dieser eigenartigen Kombination von Eiweißbausteinen in verschiedenen Organismen und deren Organen und über sein Auftreten unter bestimmten biologischen Verhältnissen versprechen prinzipiell wichtige Aufschlüsse und sind im hiesigen Institut bereits in Angriff genommen. ${ }^{1}$ ) -

1) Siehe die folgende Abhandlung von Frl. Dr. Krasnosselski. 


\section{Spezieller Teil.}

I. Über das Scombrin

von A. Kossel.

$23 \mathrm{~g}$ lufttrockenes Scombrinsulfat wurde mit einer Mischung von $75 \mathrm{~g}$ konzentrierter Schwefelsäure und $150 \mathrm{ccm}$ Wasser 6 Stunden am Rückflußkühler gekocht. Die Untersuchung auf die Verteilung des Stickstoffs unter den Spaltungsprodukten wurde in früher beschriebener Weise ${ }^{1}$ ) ausgeführt. Das Arginin wurde aus dem Stickstoffgehalt der *Argininfraktion» berechnet. Durch einen besonderen Versuch überzeugte ich mich von der Abwesenheit des freien Guanidins. Nach Entfernung des Arginins durch Pikrolonsäure war weder mit Pikrinsäure ${ }^{2}$ ) noch mit Benzolsulfochlorid ${ }^{3}$ ) Guanidin nachweisbar.

Der in Alkohol unlösliche Teil der Monoamidosäuren bestand, wie die Elementaranalyse zeigte, nicht aus reinem Alanin, sondern enthielt noch kohlenstoffreichere Beimengungen. Die Ergebnisse der Untersuchungen sind in folgender Tabelle zusammengestellt, welche eine recht bemerkenswerte Übereinstimmung des Scombrins und Salmins erweist.

Prozente des Gesamtstickstoffs.

\begin{tabular}{l|c|c}
\hline & Scombrin & Salmin ${ }^{1}$ ) \\
\hline Argininfraktion . . . . . . . . & 88,8 & 89,2 \\
In Alkohol unlöslicher Teil . . & 6,8 & 4,9 \\
In Alkohol löslicher Teil . . . . & 3,8 & 4,3 \\
Nachweisbarer Verlust . . . . . & 0,6 & 1,6
\end{tabular}

Der in Alkohol lösliche Teil ist auf Prolin zu beziehen, welches nach den früheren in Gemeinschaft mit H. D. Dakin ausgeführten ${ }^{4}$ ) Untersuchungen unter den Spaltungsprodukten des Scombrins auftritt, der in Alkohol unlösliche Teil zum Teil auf Alanin. Während wir aber in dem früher untersuchten Scombrin-

1) A. Kossel und H. D. Dakin, Diese Zeitschrift, Bd. XLI, S. 411.

2) Fr. Kutscher und J. Otori, Diese Zeitschrift, Bd. XLIII, S. 93.

$\left.{ }^{8}\right)$ D. Ackermann, Diese Zeitschrift, Bd. XLVII, S. 366.

4) Diese Zeitschrift, Bd. XLIV, S. 345. 
präparat neben den genannten Körpern keine anderen Amidosäuren auffinden konnten, zeigte sich hier, wie schon erwähnt, eine kohlenstoffreichere Substanz als Begleiterin des Alanins.

\section{Beitrag zur Kenntnis der Protone ${ }^{1}$ ) von Harold Pringle.}

Meine Untersuchungen sollten das Verhältnis der Protamine zu den Protonen klarlegen, indem sie die Zusammensetzung der Protone von neuem feststellten. Als Ausgangsmaterial wählte ich das Clupein, welches am leichtesten in größerer Menge zu erhalten ist. Das Clupeinsulfat wurde nach dem von A. Kossel früher angegebenen Verfahren ${ }^{2}$ ) dargestellt und gereinigt und zum Teil nach M. Goto ${ }^{3}$ ) in die Kupfersulfatverbindung übergeführt. Als "Argininstickstoff » wird in allen Fällen der Stickstoff der durch das Silberbarytverfahren fällbaren Substanzen bezeichnet.

Versuch 1. Bestimmung des Argininstickstoffs im Clupein. $4 \mathrm{~g}$ Clupeinsulfat wird eine halbe Stunde mit frisch bereitetem Kupferoxydhydrat gekocht, filtriert, das Filtrat im Vakuum auf ein geringes Volumen gebracht und mit Alkohol gefällt. Der Niederschlag wird mit Schwefelwasserstoff zerlegt, die Lösung eingeengt und 14 Stunden mit Schwefelsäure (1 Volum konz. Schwefelsäure mit 2 Volumen Wasser) gekocht. Das Verhältnis des Argininstickstoffs zum Gesamtstickstoff betrug 89,1:100.

Versuch 2. Derselbe Versuch wiederholt. Menge des Argininstickstoffs im Vergleich zum Gesamtstickstoff 88,7:100.

Versuch 3. Derselbe Versuch unter Anwendung konzentrierterer Schwefelsäure wiederholt. Hierbei war die Zersetzung zu weit vorgeschritten, da eine geringe Menge Ammoniak nachweisbar war. Nichtsdestoweniger war die Argininmenge fast die gleiche wie in Versuch 1 und 2. Der Argininstickstoff betrug $87,9 \%$ des Ganzen.

Versuch 4. Bestimmung des Argininstickstoffs im Proton. Zur Darstellung von Proton (Clupeon) erhitzte ich $7 \mathrm{~g}$ Clupein-

1) Die Versuche wurden mit Hilfe eines Stipendiums von der Royal Society of London ausgeführt.

2) Diese Zeitschrift, Bd. XXII, S. 176, Bd. XXV, S. 165.

$\left.{ }^{3}\right)$ Diese Zeitschrift, Bd. XXXVII, S. 94. 
sulfat eine halbe Stunde mit 10-volumprozentiger Schwefelsäure. Die Lösung gab jetzt nach dem Zusatz von etwas überschüssigem Ammoniak keinen Niederschlag mit Wittepepton, enthielt also kein Protamin mehr. Ich befreite nunmehr die Flüssigkeit durch Barytwasser von der Schwefelsäure und leitete nach dem Filtrieren zur Entfernung des Baryts Kohlensäure ein. Nachdem das Baryumcarbonat durch Eindampfen zur Trockne und Aufnehmen mit Wasser völlig entfernt war, wurde die Lösung zur Trockne gebracht.

Die weitere Reinigung bewirkte ich nach einem von Herrn Professor A. Kossel angegebenen Verfahren, welches auf folgendem Verhalten des Arginins beruht. Versetzt man eine Lösung von Arginin in Eisessig mit alkoholischer Lösung von Pikrinsäure, so entsteht ein Niederschlag, welcher sich im Überschuß von Pikrinsäure wieder löst. Behandelt man Proton, peptonartige Stoffe oder "Albumosen» in gleicher Weise, so bleibt der durch Pikrinsäure entstandene Niederschlag auch bei $\mathrm{Zu}-$ satz des überschüssigen Fällungsmittels größtenteils bestehen. Es ist auf diese Weise möglich, Proton oder pepton- und albumosenartige Substanzen von beigemengtem Arginin zu trennen.

Demgemäß löste ich das Proton in Eisessig, fällte die Lösung mit überschüssiger alkoholischer Pikrinsäure und zersetzte den erhaltenen Niederschlag mit wässeriger Schwefelsäure. Die Pikrinsäure wurde sodann durch Ausschütteln mit Äther entfernt und die schwefelsäurehaltige Lösung des so gereinigten Protons in der Siedehitze gespalten. Die Menge des Argininstickstoffs betrug 88,12\%.

Versuch 5. Bestimmung des Argininstickstoffs in verschiedenen Fraktionen des Protons. $27 \mathrm{~g}$ Clupeinsulfat wurde wie im vorigen Versuch in Proton übergeführt, durch Baryt von Schwefelsäure und durch Kohlensäure vom Baryt völlig befreit. Der eingedampfte Rückstand wurde in Eisessig gelöst und nach obigem Verfahren mit alkoholischer Pikrinsäure gefällt. Diesen Niederschlag bezeichne ich als Fraktion I.

Das pikrinsäurehaltige Filtrat wurde verdunstet, der Rückstand mit verdünnter Schwefelsäure aufgenommen und mit Äther von Pikrinsäure befreit. Nunmehr fällte ich die Lösung 
mit dem von A. Kossel zur Gewinnung des Arginins angegebenen Silberbarytverfahren. ${ }^{1}$ ) Durch dies Verfahren werden bekanntlich ${ }^{2}$ ) auch Protone und ähnliche Verbindungen niedergeschlagen. Wäre bei der Hydrolyse des Protamins, welche zur Darstellung des Protons diente, Arginin oder eine argininreichere organische Verbindung abgespalten, so hätte sich diese in dem Silberniederschlag befinden müssen. Der durch Silbersulfat und Baryt erzeugte Niederschlag wurde abfiltriert und durch Schwefelwasserstoff bei Gegenwart von Schwefelsäure zersetzt, die filtrierte Flüssigkeit von Schwefelsäure befreit und behufs weiterer Fraktionierung bei neutraler Reaktion mit Natriumpikrat gefällt. Die in diesem Niederschlag enthaltene Substanz bezeichne ich als Fraktion II, sie wurde für die weitere Verarbeitung durch Äther von der Pikrinsäure befreit. Die von Fraktion II abfiltrierte Flüssigkeit wurde mit Schwefelsäure angesäuert und von neuem mit Natriumpikrat versetzt. Der in saurer Lösung erzeugte Niederschlag enthielt denjenigen Anteil des Protons, welcher als Fraktion III bezeichnet wurde.

Fraktion I wurde nach Entfernung der Pikrinsäure mit Hilfe des Silberbarytverfahrens niedergeschlagen und die vom Silber befreite schwefelsaure Lösung des Niederschlages in zwei Teile geteilt. Der eine Teil (a) wurde direkt mit Schwefelsäure gespalten und quantitativ auf Arginin untersucht.

Der zweite Teil (b) wurde zunächst in die Kupfersulfatverbindung übergeführt, diese mit Alkohol gefällt, mit Schwefelwasserstoff zersetzt und mit Schwefelsäure gespalten. Der Arginingehalt beider Teile war der gleiche, er betrug in Prozenten des Gesamtstickstoffs.

$$
\begin{array}{ccc}
\text { Fraktion } & \text { I a } & 87,6 \\
» & \text { Ib } & 87,2
\end{array}
$$

Fraktion II ergab für den Argininstickstoff $88,8 \%$

$$
\text { " III" " } " 87,0 \% \text {. }
$$

Der Stickstoff ist somit in allen untersuchten Fraktionen in gleichem Verhältnis auf das Arginin und die Monoamidosäuren verteilt, die Unterschiede im Gehalt an Argininstickstoff

1) Diese Zeitschrift, Bd. XXV, S. 179.

2) Diese Zeitschrift, Bd. XLII, S. 185. 
erreichen nicht $2 \%$ des Gesamtstickstoffs. Fast die gleichen Zahlen werden auch für die ursprünglichen Protamine gefunden.

In Fraktion Ib des Versuchs 5 untersuchte ich die Monoamidosäuren und fand folgende Zahlen.

In Alkohol löslich (Prolin) $5,9 \%$

In Alkohol unlöslich (Serin, Alanin, Amidovaleriansäure) $5,6 \%$

Davon in Methylalkohol löslich

$4,0 \%$

In Methylalkohol unlöslich $1,6 \%$

Zur Feststellung des Stickstoffs der in Alkohol löslichen Substanz verfuhr ich in der von A. Kossel und H. D. Dakin früher angegebenen Weise. Diese Zahlen weichen von den im Clupein gefundenen ab, doch ist das Analysenmaterial für weitere Schlußfolgerungen noch nicht ausreichend.

\section{III. Über das Histon}

von A. Kossel.

Überläßt man Histon aus Thymus oder aus Vogelblutkörperchen oder aus Fischtestikeln (Gadus, Centrophorus) der mehrtägigen Einwirkung von Pepsinsalzsäure, so verschwindet allmählich die charakteristische Ammoniakreaktion und es bildet sich ein Umwandlungsprodukt, welches aus der neutralisierten Lösung durch Natriumpikrat ausgefällt werden kann. Dieser Niederschlag läßt sich unter Beobachtung bestimmter Maßregeln leicht abfiltrieren und in erheblicher Menge gewinnen. Er enthält einen pepton- oder albumoseartigen Körper, welchen ich als Histopepton bezeichnen will. Verdünnte Mineralsäure führt bei Wasserbadtemperatur dieselbe Umwandlung herbei.

Bei solchen Eiweißkörpern, welche nicht den Charakter der Histone tragen (z. B. Fibrin, Syntonin, Leim, Globin, 1) Casein) habe ich diesen Niederschlag bisher nicht in der für die Histone charakteristischen Weise erhalten, doch muß ich ausdrücklich bemerken, daß die Untersuchungen hierüber noch nicht abgeschlossen sind.

Ebenso wie das Histopepton (oder die Histopeptone) werden auch die Protamine durch Natriumpikrat bei neutraler Reaktion der Flüssigkeit gefällt. Doch lassen sich die Protamine des Salmin-, Cyclopterin- und Sturin-Typus leicht vom Histopepton

1) Das Globin ist von einigen Autoren ohne hinreichenden Grund zu den Histonen gerechnet worden. Es gibt die Reaktionen der Histone nicht. 
unterscheiden, da sie durch Eiweiß in neutraler oder ammoniakalischer Lösung gefällt werden; das Histopepton hingegen zeigt diese Fällung nicht.

Verarbeitung der Verdauungsprodukte des Histons.

Darstellung des Histopeptons. $80 \mathrm{~g}$ Histon aus Kalbsthymus wurden mit $1 \mathrm{~g}$ käuflichem Pepsin und $1500 \mathrm{ccm} 0,8 \%$ iger Salzsäure 10 Tage im Brutschrank digeriert, die Lösung nach der Neutralisation und Filtration mit Natriumpikrat im Schütteltrichter gefällt und zur Abscheidung des Niederschlages mit Äther geschüttelt. Der Niederschlag wird abgesaugt, mit einer wässerigen Lösung von Natriumpikrat ausgewaschen und durch Äther unter Zusatz von Schwefelsäure von der Pikrinsäure befreit. Aus der schwefelsauren Lösung wird das Sulfat des Histopeptons durch Alkohol herausgefällt.

Eines der analysierten Präparate (Präp. A) war in dieser Weise dargestellt worden, ein zweites (Präp. B) war noch einer weiteren Reinigung unterworfen, indem es nach Entfernung der Schwefelsäure durch das Silbernitratbarytverfahren niedergeschlagen wurde. ${ }^{1}$ )

Der Silberniederschlag wird nach dem Auswaschen durch Schwefelwasserstoff bei Gegenwart von etwas Schwefelsäure vom Silber befreit und die Lösung nach Entfernung des Schwefelwasserstoffs mit Alkohol gefällt. Durch mehrmalige Wiederholung dieses Verfahrens und durch Entfärbung mit Tierkohle gelingt es, das Sulfat des Histopeptons als weißes Pulver zu gewinnen, dessen wässerige Lösung nur eine schwach gelbe Farbe besitzt. Aus $80 \mathrm{~g}$ lufttrocknem Histon erhielt ich auf

1) Diese von mir ursprünglich für die Darstellung des Arginins angegebene Fällungsmethode ist bereits auf verschiedene Körper angewandt worden und hat bei der Isolierung des Histidins (A. Kossel und F. Kutscher), des Carnosins (Wl. v. Gulewitsch), des Thymins, Cytosins und Kreatinins (F. Kutscher) usw. gute Dienste geleistet. Nach meinen in Gemeinschaft mit H. D. Dakin ausgeführten Untersuchungen kann es auch zur Isolierung von Protonen und Protaminen dienen. Ein Teil der Peptone und Albumosen wird ebenfalls durch dies Verfahren gefällt, worüber demnächst nähere Mitteilungen erfolgen sollen. 
diese Weise 16,5 g Histopeptonsulfat; Verluste waren bei dieser Darstellung nicht $\mathrm{zu}$ vermeiden.

Darstellung der übrigen Verdauungsprodukte. Die Fällung durch Natriumpikrat ist, zumal bei Gegenwart von Neutralsalzen, keine vollständige. Ein Teil des Histopeptons bleibt neben anderen durch die Pepsinverdauung gebildeten Produkten in der Lösung. Zur Gewinnung dieses Restes von Histopepton und der übrigen Verdauungsprodukte wurde das Filtrat von Pikrinsäure befreit, bei neutraler Lösung eingedampft, sodann durch Sättigung mit Zinksulfat ausgefällt, filtriert und mit gesättigter Lösung von Zinksulfat ausgewaschen. Der Niederschlag wurde nach Entfernung des Zinks und der Schwefelsäure mit Alkohol gefällt und es wurde auf diese Weise eine nicht unbeträchtliche Menge eines Körpers dargestellt, welcher sich ähnlich wie Histopepton verhielt.

Auch in dem durch Zinksulfat nicht gefällten Anteil waren Körper von peptonartigem Charakter vorhanden, welche nach Entfernung des Zinks durch Phosphorwolframsäure gefällt wurden. Nach Entfernung der Phosphorwolframsäure wurde dieses Peptongemenge ebenfalls mit dem Silberbarytverfahren in zwei Fraktionen zerlegt. Die durch Silber nicht fällbare Fraktion wurde zur Entfernung anorganischer Stoffe nochmals mit Phosphorwolframsäure gefällt und die fällbaren Stoffe nach Entfernung der Phosphorwolframsäure durch fraktionierte Fällung mit Alkohol und Äther möglichst gereinigt.

Auf diese Weise wurde eine in warmem Methylalkohol völlig lösliche, durch Äther aus dieser Lösung fällbare Substanz erhalten, welche sich durch ihre Reaktionen mit Sicherheit vom Histopepton unterscheiden ließ. Dieselbe gab in neutraler Lösung keine Fällung mit Natriumpikrat, auch war die methylalkoholische Lösung nicht durch alkoholische Pikrinsäure fällbar. Jedoch gab dieser Körper ebenso wenig wie das Histopepton die Glyoxylsäurereaktion auf Tryptophan und lieferte bei der Säurespaltung kein Ammoniak. Die Millonsche Reaktion und die Biuretprobe waren positiv. Diese Substanz ist weiter unten als Präparat $\mathrm{G}$ bezeichnet worden. 
Zusammensetzung, Reaktionen und Spaltungsprodukte der aus Histon gewonnenen Körper.

Eigenschaften des Histopeptons. Das Sulfat des Histopeptons ist in Wasser leicht löslich, die Lösung reagiert sauer, gibt mit Ferrocyankalium weder in neutraler noch in saurer Lösung einen Niederschlag. Protamin vom Salmin- oder Sturin-Typus konnten also diesem Niederschlag nicht beigemengt sein, ebenso wenig Protalbumose. Auch Kupfersulfat ruft keine Fällung hervor.

Das Sulfat des Histopeptons (Präp. B) enthielt 14,09\% Schwefelsäure und 17,16\% N (Kjeldahl). Hieraus berechnet sich für die schwefelsäurefreie Substanz ein Stickstoffgehalt von 19,98\%. Die Substanz war fast aschefrei (ungefähr $0,1 \%$ Asche).

Das Pikrat ist in heißem Wasser löslich und fältt beim Erkalten in öligen Tropfen aus, ein gewisser Überschuß von Natriumpikrat vermindert die Löslichkeit, Gegenwart von Kochsalz vermehrt sie und man kann das Pikrat durch Kochsalz zur Auflösung bringen.

Die Lösung des Histopeptonsulfats gibt die Biuretprobe und die Millonsche Reaktion, jedoch nicht die Glyoxylsäurereaktion von Hopkins und Cole, auch enthält sie keinen durch Kochen mit alkalischer Bleilösung nachweisbaren unoxydierten Schwefel. Beim anhaltenden Kochen mit Säuren färbt sie sich nur schwach bräunlich und gibt keinen Niederschlag von dunkelgefärbten Huminsubstanzen, auch wird hierbei kein Ammoniak abgespalten.

Schmilzt man $1 \mathrm{~g}$ Histopeptonsulfat mit Kalihydrat bei $180^{\circ}$, so erhält man zwar Indolgeruch, doch keine Farbenreaktion auf Indol, während man dieselbe mit Leichtigkeit bei demselben Verfahren mit 0,1 $\mathrm{g}$ Wittepepton erhalten kann. ${ }^{1}$ ) Die Substanz liefert also nur äußerst geringe Spuren von Indol.

1) Der Nachweis der lndolgruppe in kleineren Mengen Eiweiß kann bequem in folgender Weise geführt werden. In einem Reagensglas von ungefähr $15 \mathrm{~cm}$ Länge und $3 \mathrm{~cm}$ lichtem Durchmesser befindet sich ein zylindrisches Gefäß aus Nickel, dessen Durchmesser.so groß ist, daß es der Glaswand dicht anliegt, und dessen Länge etwa $7 \mathrm{~cm}$ beträgt, das Nickelgefäß enthält eine angefeuchtete Mischung des zu untersuchenden Eiweißkörpers 
Methode der quantitativen Spaltung des Histopeptons. Im wesentlichen benutzte ich das von mir in Gemeinschaft mit F. Kutscher beschriebene Verfahren. Doch wurden folgende Modifikationen angebracht.

Zur Trennung von Arginin und Histidin wurde das von uns beschriebene Silberverfahren benutzt, jedoch wurde die' Neutralisation nicht durch Barytwasser unter Kontrolle der ammoniakalischen Silberlösung, sondern durch Baryumcarbonat bei W'asserbadtemperatur bewirkt.

Die vollständige Ausfällung des Histidins läßt sich leicht nachweisen, wenn man eine Probe des aus dem Filtrat dargestellten Arginins mit Hilfe der Diazobenzolsulfosäurereaktion ${ }^{2}$ ) prüft. Die geringsten Spuren von Histidin bewirken einen positiven Ausfall dieser Reaktion, während das Arginin die Reaktion nicht gibt.

Der durch Baryumcarbonat ausgefällte Histidinsilberniederschlag wird durch Schwefelwasserstoff bei Gegenwart von Schwefelsäure zerlegt, vom Schwefelsilber abfiltriert und letzteres mit siedendem Wasser völlig erschöpft. Die vereinigten Filtrate und Waschwasser wurden nach dem Verdunsten des Schwefelwasserstoffs zur Entfernung der Schwefelsäure mit überschüssigem Barytwasser versetzt und durch Kohlensäure vom Baryt befreit. Die völlig barytfreie Lösung wurde auf $500 \mathrm{ccm}$ aufgefüllt und eine gemessene Menge (z. B. $50 \mathrm{ccm})$ zur Stickstoffbestimmung nach Kjeldahl entnommen. Der übrig bleibende Teil diente zur Überführung des Histidins in das

mit dem zehnfachen Gewicht Ätzkali. Das Glasrohr taucht in ein Ölbad und wird 1 1/2 -2 Stunden bei einer Temperatur des Bades von $260-270^{\circ}$ erhitzt. Die Schmelze wird sodann in Wasser gelöst, die wässerige Lösung im Extraktionsapparat mit Äther erschöpft und der Äther bei gelinder Temperatur auf dem Wasserbade verdunstet. Hierbei bemerkt man eventuell Indolgeruch. Der Verdampfungsrückstand dient zur Anstellung der Indolreaktionen, von denen die «holerarot-Reaktion in der von Salkowski angegebenen Form besonders empfindlich ist (cf. Salkowski, Virchows Archiv, Bd. CX, S. 366 [1889]).

Will man die bei der Schmelzung entwickelten flüchtigen Stoffe auffangen, so fügt man einen doppelt durchbohrten, mit Glasröhren versehenen Kork in das Reagenzrohr und treibt die Destillationsprodukte durch einen Wasserstoffstrom in eine Vorlage über.

1) cf. Pauly, Diese Zeitschrift, Bd. XLII, S. 508. 
Pikrolonat. Die für diesen Zweck erforderliche Menge Pikrolonsäure wurde in der Weise bemessen, daß auf 3 Atome Stickstoff der Lösung etwas mehr als 1 Molekül Pikrolonsäure in Alkohol gelöst zu der Lösung des freien Histidins hinzugefügt wurde.

Nach dem Eindampfen auf ein geringes Volumen scheidet sich ein reichlicher krystallinischer Niederschlag ab, welcher abfiltriert und in $500 \mathrm{ccm}$ siedendem Wasser gelöst wurde. Beim Erkalten fällt der größte Teil des Histidinpikrolonats krystallinisch aus. Derselbe wurde durch ein gewogenes Filter abfiltriert, das Filter auf $15 \mathrm{ccm}$ eingedampft und der nach dem Erkalten neu entstandene Niederschlag zu dem ersten hinzufiltriert, mit wenig kaltem Wasser gewaschen und das Filter mit dem Niederschlag getrocknet und gewogen. Aus dem Pikrolonat wurde das Histidin oder der in Form von Histidin vorhandene Stickstoff nach der von Steudel angegebenen ${ }^{1}$ ) Formel

berechnet.

$$
\mathrm{C}_{10} \mathrm{H}_{8} \mathrm{~N}_{4} \mathrm{O}_{5} \cdot \mathrm{C}_{6} \mathrm{H}_{9} \mathrm{~N}_{8} \mathrm{O}_{2}
$$

Zur Bestimmung des Arginins wurde die vom Histidinsilber abfiltrierte Flüssigkeit in der früher beschriebenen Weise ${ }^{2}$ ) verarbeitet und der Stickstoffgehalt der Lösung festgestellt. Das Arginin wurde sodann in das Pikrolonat übergeführt und als solches gewogen, indem eine genau gemessene Menge der vollkommen von Baryt befreiten Lösung des Arginincarbonats mit alkoholischer Lösung von Pikrolonsäure versetzt und eingedampft wurde. Die erforderliche Menge Pikrolonsäure war nach der Stickstoff bestimmung zu berechnen, indem auf je 4 Atome Stickstoff etwas mehr als ein Molekül Pikrolonsäure hinzugefügt wurde. Das nach längerem Stehen auskrystallisierte Argininpikrolonat wurde auf gewogenem Filter gesammelt und gewogen, aus ihm berechnet ${ }^{3}$ ) sich das Arginin nach der Formel $\mathrm{C}_{10} \mathrm{H}_{8} \mathrm{~N}_{4} \mathrm{O}_{5} \cdot \mathrm{C}_{6} \mathrm{H}_{14} \mathrm{~N}_{4} \mathrm{O}_{2}$.

Das Lysin wurde mit Phosphorwolframsäure gefällt und als Pikrat gewogen. Das Filtrat des Phosphorwolframsäure-

1) Diese Zeitschrift, Bd. XLIV, S. 158.

$\left.{ }^{2}\right)$ Diese Zeitschrift, Bd. XXXI, S. 174.

) Diese Zeitschrift, Bd. XLIV, S. 157. 
niederschlages wurde von Phosphorwolframsäure befreit und nach Entfernung des Baryts zur Trockne gebracht. Der Rückstand wurde mit siedendem Alkohol extrahiert, der Alkohol verdunstet und die zurückbleibende Masse von neuem mit Alkohol aufgenommen. Hierauf wurde der Stickstoff des alkohollöslichen Anteils nach Kjeldahl bestimmt.

Der in Alkohol unlösliche Teil wurde nach Habermann und Ehrenfeld mit einer Mischung von gleichen Teilen Eisessig und Alkohol extrahiert und der unlösliche Teil als Tyrosin in Rechnung gezogen. Weiter wurde das Gemisch der Monoamidosäuren nicht aufgelöst.

Ergebnisse der Spaltung. Folgende Tabellen zeigen die Analysenzahlen von Präparat A und B des Histopeptons. Hierzu ist $\mathrm{zu}$ bemerken, daß die für Präparat $\mathrm{B}$ erhaltenen Zahlen als die zuverlässigeren zu betrachten sind. Denn diese Bestimmungen wurden mit einem Präparat ausgeführt, welches weitergehenden Reinigungsprozeduren unterworfen war. Auch betrug die zur Analyse verwendete Substanzmenge bei Präparat A nur 4,88, bei Präparat B 9,43 g trocknes freies Histopepton (aus dem Stickstoffgehalt berechnet). Die Wägung des Histidins und Arginins als Pikrolonat wurde nur bei Präparat B ausgeführt.

Prozente des Gesamtstickstoffs.

\begin{tabular}{l|c|c}
\hline & $\begin{array}{c}\text { Präparat } \\
\text { A }\end{array}$ & $\begin{array}{c}\text { Präparat } \\
\text { B }\end{array}$ \\
\hline Gesamtmenge . . . . . . . . . . . . . . & 100 & 100 \\
Histidinfraktion. Direkte Stickstoff bestimmung . & 3,6 & 3,7 \\
Histidin als Pikrolonat gewogen . . . . . . . & - & 4,0 \\
Argininfraktion. Direkte Stickstoff bestimmung . & 23,9 & 27,2 \\
Arginin als Pikrolonat gewogen . . . . . . . & - & 25,8 \\
Lysin als Pikrat gewogen . . . . . . . . . . & 11,3 & 17,3 \\
Durch Phosphorwolframsäure nicht fällbar . . & 26,9 & 27,7 \\
Tyrosin . . . . . . . . . . . . . . . & 0,9 & 1,2 \\
\end{tabular}

Zum Vergleich verweise ich auf die oben S. 307 gegebene Zusammenstellung der Verteilung des Stickstoffs in den Histonen. 
Gegenüber diesen Zahlen kommt der Darstellung der Analysenergebnisse in Gewichtsprozenten eine geringere Bedeutung zu. Immerhin mögen auch diese in folgender Tabelle (unter Annahme eines Stickstoffgehalts von 20,0\% im Histopepton berechnet) Platz finden.

Gewichtsprozente der Spaltungsprodukte.

\begin{tabular}{|c|c|c|}
\hline & Präparat A & Präparat $\mathrm{B}$ \\
\hline Histopepton . . . . . . & 100 & 100 \\
\hline Histidin . . . . . . . . & 2,7 & 3,2 \\
\hline Arginin . . . . . . & 14,9 & 16,0 \\
\hline Lysin . . . . . . . . . & 11,8 & 18,1 \\
\hline Tyrosin : . . . . & 2,3 & 2,9 \\
\hline
\end{tabular}

Es entfällt somit im Histopepton ebenso wie im Histon ungefähr der vierte Teil des Stickstoffs auf das Arginin. Ungefähr : ebenso viel findet sich im Filtrat des Phosphorwolframsäureniederschlages. Der Gehalt des Histopeptons an solchen Monoamidosäuren, welche durch Phosphorwolframsäure nicht gefällt werden, ist also im Vergleich mit sonstigen eiweißartigen Substanzen ein geringer. Auffallend hoch ist die Menge des Lysins.

Neben dem Histopepton entstehen, wie schon erwähnt, noch andere peptonartige Stoffe bei der Pepsinverdauung des Histons. Eine dieser Substanzen, die S. 316 unter der Bezeichnung . Präp. C * erwähnt worden ist, wurde einer weniger eingehenden Untersuchung unterworfen. Hierbei fanden sich 19,5 Prozent des Gesamtstickstoffs in dem Silberniederschlag (größtenteils Arginin und Histidin), 19,8\% in dem Phosphorwolframsäureniederschlag (größtenteils Lysin) und $41,7 \%$ in dem Filtrat dieses Niederschlags. Diese Substanz enthielt daher bedeutend weniger Arginin und mehr Monoamidosäuren als das Histopepton.' 